\title{
LÓGICA FUZZY NA ANÁLISE ESPACIAL DOS TEORES DE BORO E MANGANÊS NO TECIDO FOLIAR DO CAFEEIRO CONILON
}

\author{
Mariana Lima de Jesus ${ }^{1}$ \\ Abel Souza da Fonseca ${ }^{2}$ \\ Tiago da Silva Machado ${ }^{3}$ \\ Gevson Roldi Junior ${ }^{4}$ \\ Julião Soares de Souza Lima ${ }^{5}$
}

Resumo: Essa pesquisa objetivou utilizar lógica Fuzzy para mapear os teores foliares dos micronutrientes em café conilon, considerando sua variabilidade espacial. O estudo foi realizado na fazenda experimental do INCAPER, onde forma coletadas folhas recém-maduras para determinação dos teores de Mn e B. Fezse a análise de estatística descritiva e geoestatística dos teores foliares; definida a dependência espacial, foram confeccionados mapas temáticos por meio da krigagem. A partir dos mapas, determinou-se o universo de discurso de cada nutriente, seguido da construção dos conjuntos fuzzy de entrada, por meio da função trapezoidal. Com os mapas dos teores foliares realizou-se a classificação fuzzy. A lógica fuzzy permitiu visualizar as mudanças gradativas das faixas tidas como ideais para B e Mn.

Palavras-chave: Lógica difusa; Geoestatística; Análise foliar; Coffea canephora.

\footnotetext{
1 Mestrado em Produção Vegetal/Universidade Federal do Espírito Santo, Brasil. E-mail: marianaldejesus@gmail.com.

2 Doutorado em Produção Vegetal/Universidade Federal do Espírito Santo, Brasil. E-mail: abelsouzafonseca@gmail.com.

3 Graduação em Agronomia/Universidade Federal do Espírito Santo, Brasil. E-mail: tiagomachadao@hotmail.com.

4 Mestrado em Produção Vegetal/Universidade Federal do Espírito Santo, Brasil. E-mail: junior_roldi@hotmail.com.

5 Professor/Universidade Federal do Espírito Santo, Brasil. E-mail: limajss@yahoo.com.br.
} 